\title{
Biological Parts for Kluyveromyces marxianus Synthetic Biology
}

\author{
Arun S. Rajkumar ${ }^{1}$, Javier A. Varela ${ }^{1}$, Hannes Juergens ${ }^{2}$, Jean-Marc G. Daran ${ }^{2}$ and \\ John P. Morrissey ${ }^{1 *}$
}

${ }^{1}$ School of Microbiology, Centre for Synthetic Biology and Biotechnology, Environmental Research Institute, APC Microbiome Institute, University College Cork, Cork, Ireland, ${ }^{2}$ Department of Biotechnology, Delft University of Technology, Delft, Netherlands

OPEN ACCESS

Edited by:

Rodrigo Ledesma-Amaro, Imperial College London,

United Kingdom

Reviewed by:

Dae-Hee Lee,

Korea Research Institute of Bioscience and Biotechnology

(KRIBB), South Korea

Hyun Ah Kang,

Chung-Ang University, South Korea

${ }^{*}$ Correspondence:

John P. Morrissey

j.morrissey@ucc.ie

Specialty section:

This article was submitted to

Synthetic Biology,

a section of the journal

Frontiers in Bioengineering and

Biotechnology

Received: 25 November 2018

Accepted: 16 April 2019

Published: 07 May 2019

Citation:

Rajkumar AS, Varela JA, Juergens $H$, Daran JM and Morrissey JP (2019) Biological Parts for Kluyveromyces marxianus Synthetic Biology. Front. Bioeng. Biotechnol. 7:97. doi: 10.3389/fbioe.2019.00097
Kluyveromyces marxianus is a non-conventional yeast whose physiology and metabolism lends itself to diverse biotechnological applications. While the wild-type yeast is already in use for producing fragrances and fermented products, the lack of standardised tools for its genetic and metabolic engineering prevent it from being used as a next-generation cell factory for bio-based chemicals. In this paper, we bring together and characterise a set of native $K$. marxianus parts for the expression of multiple genes for metabolic engineering and synthetic biology. All parts are cloned and stored according to the MoClo/Yeast Tool Kit standard for quick sharing and rapid construction. Using available genomic and transcriptomic data, we have selected promoters and terminators to fine-tune constitutive and inducible gene expression. The collection includes a number of known centromeres and autonomously replication sequences (ARS). We also provide a number of chromosomal integration sites selected for efficiency or visible phenotypes for rapid screening. Finally, we provide a single-plasmid CRISPR/Cas9 platform for genome engineering and facilitated gene targeting, and rationally create auxotrophic strains to expand the common range of selection markers available to $K$. marxianus. The curated and characterised tools we have provided in this kit will serve as a base to efficiently build next-generation cell factories from this alternative yeast. Plasmids containing all parts are available at Addgene for public distribution.

Keywords: Kluyveromyces, synthetic biology, metabolic engineering, genome engineering, yeast

\section{INTRODUCTION}

Cell factories can serve as the basis of a new bio-based economy based on the sustainable production of fine chemicals, pharmaceuticals, nutraceuticals, and biofuels from engineered or native microbes. As of now, the most commonly-used eukaryotic cell factory is baker's yeast Saccharomyces cerevisiae. An ease of genetic manipulation, along with a wealth of genomic, genetic and biochemical knowledge, have made it able to produce a diverse range of compounds with S. cerevisiae from simple or economical feedstocks. However, non-Saccharomyces yeasts can provide several advantages over $S$. cerevisiae for building cell factories as they often possess desirable tolerances or metabolic traits that would otherwise need to be extensively engineered into baker's yeast (Wagner and Alper, 2016). In general, such yeasts have had niche applications in biotechnology but are being developed to be next-generation cell factories. Kluyveromyces marxianus is one such alternative yeast. While thermotolerant, fast-growing and able to use various carbon and nitrogen sources, it broadly has the same nutritional requirements and culture 
techniques as $S$. cerevisiae. Its natural strain diversity allows a wide number of phenotypes and gene variants to be exploited and combined to create an optimal cell factory chassis, and as a broadly Crabtree-negative species, it does not need to have metabolism routed away from ethanol production. While it has applications in the production of aroma compounds, fermented foods, and secreted enzymes (Morrissey et al., 2015; Gombert et al., 2016), K. marxianus is yet to be established as a metabolic engineering platform. Obstacles to such development include inefficient and random native gene targeting (hindering the stable expression of integrated heterologous genes), limited knowledge of its biochemistry and genetics, and a lack of standardised regulatory parts and expression systems on the level of baker's yeast. While such tools are starting to be developed, it still lacks the well-defined sets of regulatory elements to precisely control gene expression one uses in S. cerevisiae. As of now, individual parts are either selected from the native genome based on orthologues of genes of common parts from S. cerevisiae (Lee et al., 2013), or from other yeasts altogether (Chang et al., 2013), precluding the advantages of using K. marxianus' environmental triggers to fine-tune gene expression. Taken together, less than 20 native regulatory parts are currently in use for metabolic engineering (Bergkamp et al., 1993; Ball et al., 1999; Yang et al., 2015a; Gombert et al., 2016).

Kluyveromyces marxianus-specific techniques exist for the efficient in vivo assembly of large multigene constructs (Chang et al., 2012), and in conjunction with CRISPR/Cas9 (Löbs et al., 2017; Nambu-Nishida et al., 2017; Cernak et al., 2018) can allow us to specifically edit a genome, or efficiently target chromosmal integrations. Nonetheless in vivo assembly as it stands does not eliminate non-specific integrations of incomplete parts of the assembly. To further sidestep this problem, the MoClo standard, based on Golden Gate assembly, allows the efficient hierarchical in vitro assembly of multigene constructs either on episomal or integrative vectors for such purposes (Weber et al., 2011). It has been adapted for synthetic biology in diverse organisms, and is efficient enough to circumvent in vivo assembly. One variant of MoClo, the Yeast Toolkit (YTK), collects a number of well-characterised parts for S. cerevisiae (Lee et al., 2015). The YTK has 8 general classes of parts, defined by the 5', and 3' overhangs used for Golden Gate assembly, which allow directional cloning. Taken together, the parts of the original YTK and the system itself allow for the versatile construction of vectors for $S$. cerevisiae with several selection markers and integration sites of choice if needed.

The YTK's MoClo approach also sets up three tiers of plasmids for storage or use (Figure 1B). Level I plasmids correspond to part plasmids. A BsmBI site, and a BsaI site that after digestion will generate overhangs specific to that part type, flank each functional part. The BsmBI sites, in turn, are used to clone new parts into the entry vector YTK001 by Golden Gate assembly with that enzyme. At level II, level I plasmids are assembled together with BsaI to create gene expression cassettes or transcriptional units (TUs). Assembling TUs includes flanking them with synthetic and directional connector sequences which allow the construction of level III, plasmids. Here, multiple TUs are assembled together into a multigene expression or integrative vector, again with BsmBI based on unique overhangs present in the connectors.

The YTK, either with its standard or simply by using its parts collection, has since been used in diverse metabolic engineering and synthetic biology applications (Feng et al., 2015; Awan et al., 2017; Denby et al., 2018), and has already been adopted when cloning parts for Pichia pastoris (Obst et al., 2017). In this paper we present a collection of $K$. marxianus-specific parts and vectors, cloned to the YTK standard, for the genetic and metabolic engineering of this yeast. Over 30 constitutive and inducible promoters, terminators and centromeres and autonomously replicating sequence (ARS) elements have been selected from existing gene expression data and characterised as part of this collection. We have also identified a number of integration sites for the integration of single- and multi-gene constructs and have tested the best means to eliminate random integration. Taken together with existing YTK parts, our parts collection is a valuable set of tools for researchers working with $K$. marxianus as a cell factory.

\section{MATERIALS AND METHODS}

\section{Strains and Cultivation}

Kluyveromyces marxianus strain CBS6556 (Westerdijk Fungal Biodiversity Institute, Utrecht, The Netherlands) was used for parts, unless specified otherwise. For part characterization and mutant creation, we used NBRC1777 [Biological Resource Centre, NITE (NBRC), Tokyo, Japan]. All strains used in this study are listed in Table 1 . Yeast cultures were routinely grown in YPD broth (1\% yeast extract, $2 \%$ peptone, $2 \%$ glucose) at $30^{\circ} \mathrm{C}$. For promoter and terminator characterization, yeast was grown in synthetic drop-out medium without uracil [SD-ura; $0.17 \%$ yeast nitrogen base without amino acids or ammonium sulphate (Formedium, Hunstanton, UK), 0.5\% ammonium sulphate and $0.19 \%$ SC-ura (Formedium)] containing 2\% glucose or an alternate carbon source. For transformation of auxotrophic strains, yeast was grown in synthetic drop-out (SD) medium containing $2 \%$ glucose and lacking the appropriate nutrients (Formedium). For fermentation experiments, strains were cultured in minimal medium (MM) with $2 \%$ glucose (Fonseca et al., 2007). When needed, G418 or hygromycin (Fisher Scientific, Dublin, Ireland) was added to a concentration of $200 \mathrm{mg} \mathrm{L}^{-1}$ for selection or $150 \mathrm{mg} \mathrm{L}^{-1}$ for maintenance. Bacterial transformations used E.coli $\mathrm{DH} 5 \alpha$ grown in LB medium ( $1 \% \mathrm{NaCl}, 1 \%$ peptone, $0.5 \%$ yeast extract) supplemented with the appropriate antibiotics $\left(100 \mathrm{mg} \mathrm{L}^{-1}\right.$ ampicillin, $50 \mathrm{mg} \mathrm{L}^{-1}$ chloramphenicol or $50 \mathrm{mg} \mathrm{L}^{-1}$ kanamycin).

\section{Selection and Amplification of Parts}

Native promoters, terminators, and replicating sequences were amplified from the genomic DNA of $K$. marxianus CBS6556 (Jeong et al., 2012) unless specified otherwise. The sequences in question were identified from the genome sequence data (assembly GCA 000299195.2) following in-house gene prediction (Varela et al., 2017). In general, a promoter was defined as the first 1,000 bases upstream of a gene's start codon, unless this overlapped with a neighbouring gene or other 
A

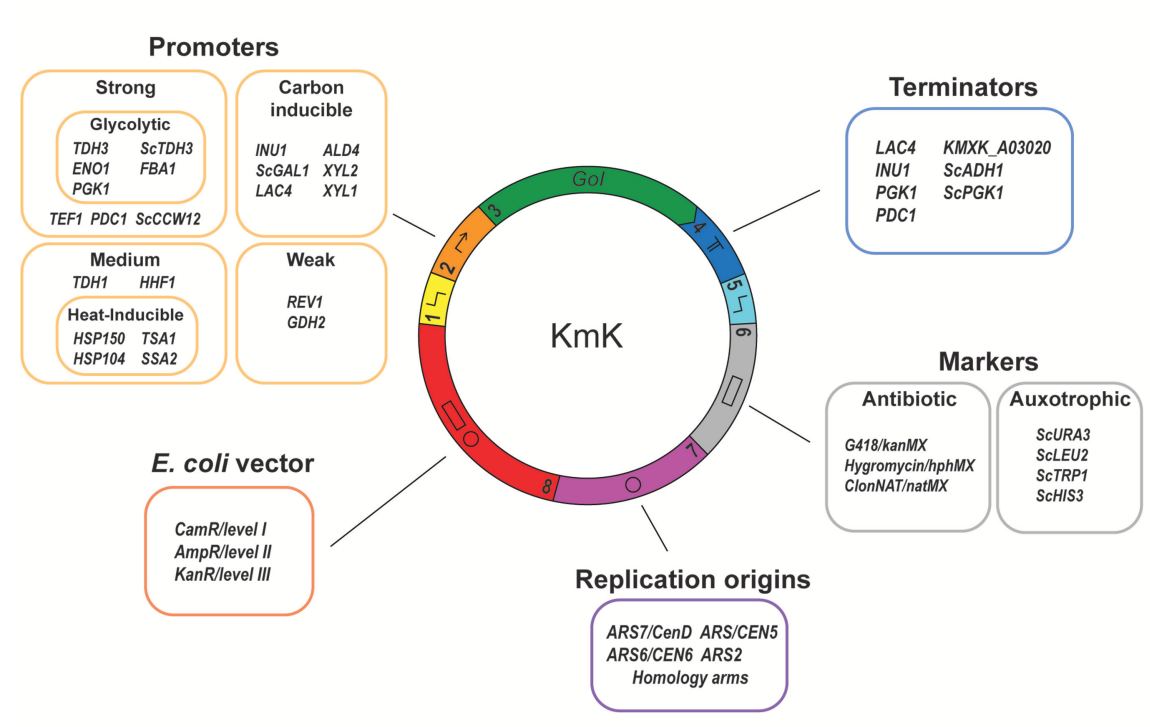

B

(1)

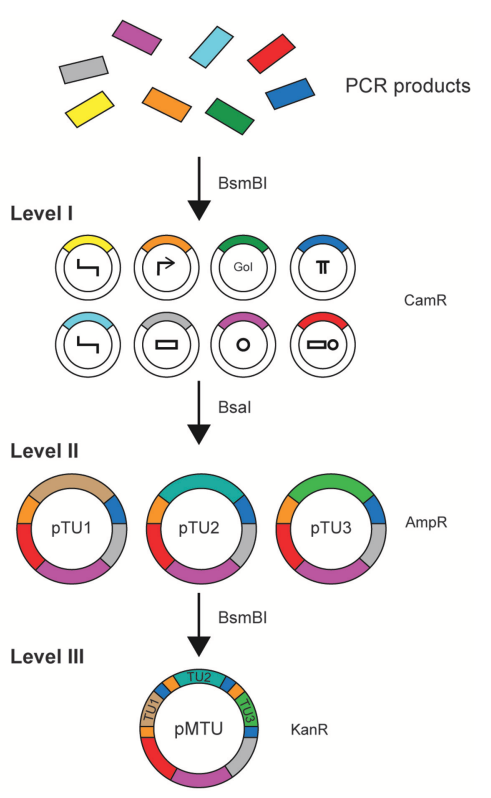

FIGURE 1 | A collection of biological parts and synthetic biology tools for Kluyveromyces marxianus. (A) The Kluyveromyces Kit (KmK) provides parts according to the Yeast Toolkit (YTK) standard to express any gene of interest (Gol) under various conditions and expression platforms. Several constitutive and inducible promoters allow precise expression of a Gol under different conditions specific to $K$. marxianus, and terminator choice further fine-tunes gene expression. A number of metabolic and antibiotic markers can be used in wild-type yeast, or in auxotrophs generated by the CRISPR/Cas9 system provided. Finally, a number of species-specific origins and integration homology arms allow the expression of the Gol on a stable plasmid or as an integration cassette. (B) Hierarchy of YTK assemblies. Alternating the type IIS enzymes between assemblies allows construction of different plasmid "levels." Starting from amplified PCR products, parts are cloned into level I plasmids for storage. At the time of cloning, they are given overhangs corresponding to the numbered parts used to build expression systems. From there, individual transcriptional units are built from level I plasmids either for use or for storage; these are level II plasmids. Finally, multiple TU-bearing level II plasmids can be combined to create multi-TU level III plasmids that are either episomal or integrative vectors. The use of different bacterial markers at each level allows us to use the previous level's plasmids directly for assembly.

TABLE 1 | List of $K$. marxianus strains used in this paper.

\begin{tabular}{llll}
\hline Strain name & Source & Genotype & Comments \\
\hline CBS6556 & $\begin{array}{l}\text { Westerdijk Fungal Biodiversity } \\
\text { Institute, Netherlands }\end{array}$ & Wild-type & \\
NBRC1777 & NITE Biological Resource Center & Wild-type & \\
YBL001 & This study & NBRC1777 yku80-1 & Frameshift in codon 346 (double basepair deletion) \\
KmASR.005 & This study & NBRC1777 dn/4-1 & Frameshift in codon 11 (single base pair insertion) \\
YBL003 & This study & NBRC1777 nej1-1 & Frameshift in codon 61 (single base pair deletion) \\
KmASR.006 & This study & NBRC1777 ura3-1 & Frameshift in codon 139 (double base pair deletion) \\
KmASR.007 & This study & NBRC1777 his3-1 & Frameshift in codon 71 (single base pair insertion) \\
KmASR.008 & This study & NBRC1777 ura3-1 his3-2 & HIS3: Frameshift in codon 126 of HIS3 (double base pair deletion) \\
KmASR.022 & This study & NBRC1777 leu2-1 & Frameshift across codons 124 and 125 double base pair deletion) \\
KmASR.023 & This study & NBRC1777 ura3-1 leu2-1 & LEU2: Frameshift across codons 124 and 125 (double base pair deletion) \\
KmASR.024 & This study & NBRC1777 his3-1 leu2-1 & LEU2: Frameshift across codons 124 and 125 (double base pair deletion) \\
KmASR.025 & This study & NBRC1777 ura3-1 his3-2 leu2-1 & LEU2: Frameshift across codons 124 and 125 (double base pair deletion) \\
\hline
\end{tabular}

features. Terminators were similarly defined as the first $250 \mathrm{bp}$ downstream of a gene's Stop codon (Curran et al., 2013). Parts were amplified from genomic DNA using Q5 High-Fidelity Polymerase (New England Biolabs, Ipswich, UK) and purified using a GeneJet PCR clean-up kit (Fisher Scientific) and eluted in sterile water prior to use. Yeast ARS elements and centromeres were selected from the literature, and either amplified from genomic DNA or from an appropriate plasmid. All assembled part plasmids with their Addgene plasmid IDs are listed in Table S1, and primers to amplify the parts are listed in Table S2.

\section{Golden Gate Assembly}

Golden Gate assemblies were carried out as recommended (Lee et al., 2015) with minor modifications. In a typical reaction, 40 
fmol of each insert (either as an existing level I plasmid or PCR product) were combined with $20 \mathrm{fmol}$ of the plasmid containing the backbone for the final product to be assembled along with $1 \mu \mathrm{L}$ T4 DNA ligase $0.5 \mu \mathrm{L}$ each of T7 DNA ligase $\left(3,000 \mathrm{U} \mu \mathrm{L}^{-1}\right)$ and BsmBI or BsaI, $\left(10 \mathrm{U}_{\mu \mathrm{L}^{-1}}, \mathrm{NEB}\right)$ and water to a total volume of $10 \mu \mathrm{L}$.

For Golden Gate assembly the protocol was as follows: 25 cycles of digestion and ligation $\left(2 \mathrm{~min}\right.$ at $42^{\circ} \mathrm{C}$ followed by $5 \mathrm{~min}$ at $16^{\circ} \mathrm{C}$ ), followed by $10 \mathrm{~min}$ digestion at $60^{\circ} \mathrm{C}$ and $10 \mathrm{~min}$ inactivation at $80^{\circ} \mathrm{C}$. For assemblies involving BsaI, the digestion step was carried out at $37^{\circ} \mathrm{C}$ for $3 \mathrm{~min}$. When plasmids containing type IIS restriction sites that could not be removed, the final digestion and heat inactivation steps were eliminated.

Typically, half of a Golden Gate assembly was used to transform chemically competent E.coli DH5 $\alpha$. Transformants were screened by colony PCR with OneTaq Quick-Load DNA polymerase (NEB), using primers in the backbone vector flanking the insert if size permitted, or with one primer binding in the backbone and one in the insert. In the latter case, this was usually a primer used to amplify one of the component parts. For level I plasmids, colonies with the correct PCR product size had their plasmids extracted and inserts sequenced. For level II and III plasmids, PCR-positive colonies had their plasmids extracted and digested with NotI to verify the insert size All primers used to genotype strains are listed in Table S3, and maps of all the plasmids in Table S1 are included as Supplementary Material.

\section{Construction of Reporter Plasmids}

$20 \mathrm{fmol}$ of a suitable "vector" level I plasmid from the Yeast Toolkit [pYTK083; (Lee et al., 2015)] were combined with $40 \mathrm{fmol}$ each of the following "insert" level I plasmids: left and right connectors from the Yeast Toolkit (pYTK002 and pYTK067), mVenus (pYTK034), a kanMX expression marker for G418 resistance, and the appropriate $K$. marxianus promoters (P1-19), terminators (T1-5), and centromere (typically C1; Table 2). A Golden Gate assembly for a level II plasmid was then carried out as described above. In total, 8 parts were assembled and transformed into E.coli per plasmid. Assembly of the construct was verified by colony PCR with ASR_K001F and ASR_K005R as primers and subsequent restriction mapping of plasmids from PCR-positive colonies with NotI. All original YTK plasmids used for assemblies are listed in Table S4.

\section{Construction of Integrative Reporter Constructs}

For the construction of integrative reporter vectors to evaluate insertion sites, a similar 8-part Golden Gate assembly was carried out as above, except that pYTK002 was replaced with plasmids containing 850-900 bp left homology arms targeting an insertion site (I1L-I5L), and C1 was replaced with plasmids containing 850-900 bp right homology arms targeting the same insertion site (I1R-I5R). For the experiments carried out here, for the purpose of evaluating integration sites, mVenus was always under the control of the PDC1 promoter (P2) and the INU1 terminator (T1). Following assembly and transformation, transformants were screened by colony PCR with primers ASR_K001F and ASR_P002R, followed by NotI restriction digestion as above.

\section{Promoter and Terminator Characterization}

We constructed mVenus (YFP) reporter plasmids to characterise promoter and terminator strength. To minimise variations in expression due to copy number, we used centromeric plasmids with a kanMX cassette. While evaluating promoter strengths alone the reporter plasmids used the inulinase terminator in common (INU1t). In a similar manner, we used histone B promoter (HHF1pr) in common to regulate YFP expression when evaluating terminator strengths. Three hundred nanogram of reporter plasmids were transformed into $K$. marxianus by the LiOAc/PEG method (Gietz, 2014). After 48 h growth on selective medium, three transformant colonies were inoculated into $2 \mathrm{~mL}$ YPD with G418 and grown overnight at $30^{\circ} \mathrm{C}$ with $200 \mathrm{rpm}$ agitation. The following day, the cultures were diluted 100 -fold into $2 \mathrm{~mL} \mathrm{SD}$ medium with $150 \mathrm{mg} \mathrm{L}^{-1} \mathrm{G} 418$ (approximately corresponding to a starting optical density of 0.1 ) and grown at $30^{\circ} \mathrm{C}$ for $24 \mathrm{~h}$ with $200 \mathrm{rpm}$ shaking. For promoter inductions at high temperatures and xylose, overnight cultures were typically inoculated to a starting OD of $0.2-0.3$ so that a comparable cell number would be present after $24 \mathrm{~h}$ growth. The cultures were then diluted 5 to 20 -fold in identical SD medium on a 96-well microtitre plate, and YFP fluorescence measured on a Sirius HT platereader (MWG/BioTek, Winooski, USA) with excitation and emission set to 485 and $525 \mathrm{~nm}$, respectively (bandwidth $20 \mathrm{~nm}$ ). After correcting for the autofluorescence of wild-type $K$. marxianus, the signal was normalised to cell number by dividing by the OD at $600 \mathrm{~nm}$. Differences between normalised YFP values under different conditions were tested for statistical significance by a paired t-test, with $p<0.05$ taken to be significant.

For the characterization of integration sites, $2 \mu \mathrm{g}$ of integrative plasmid containing an YFP expression cassette and kanMX marker was digested with SgsI/AscI (Fisher Scientific) and transformed into yeast. The amount corresponds to approximately $400 \mathrm{fmol}$ of insertion cassette. G418-resistant colonies were screened for correct insertion at the intended locus by colony PCR and these alone were selected for YFP measurements. When the LAC4 locus was targeted, transformation plates were replica-plated onto YPGal (2\% galactose, $2 \%$ peptone, $1 \%$ yeast extract) containing $200 \mathrm{mg} \mathrm{L}^{-1}$ G418 and $40 \mathrm{mg} \mathrm{L}^{-1} \mathrm{X}-\mathrm{Gal}$ (Melford Laboratories, Ipswich, UK). The inability of disrupted LAC4 to metabolise X-Gal-and not produce a blue dye-was made use of to pre-screen transformant colonies before genotyping them.

\section{Genome Editing Using CRISPR/Cas9}

The cross-yeast CRISPR/Cas9 plasmid pUDP002 (Juergens et al., 2018) was modified to pUCC001 to allow easy cloning of new guide RNA (gRNA) targets by Golden Gate assembly. The original gRNA expression cassette of pUDP002 consisted of a target gRNA and structural element flanked by selfcleaving hammerhead and HDV ribozymes at the $5^{\prime}$ and $3^{\prime}$ ends respectively ( $\mathrm{Ng}$ and Dean, 2017). This was modified to contain a BsaI cloning site between the hammerhead ribozyme and gRNA structural element (Vyas et al., 2015). The new cassette was assembled from long oligonucleotides (Integrated DNA Technologies, USA) by annealing them in a thermocycler. 
TABLE 2 | List of parts provided in the collection.

\begin{tabular}{|c|c|c|c|c|}
\hline Part & Source & YTK part type & $\begin{array}{l}\text { Gene/ } \\
\text { systematic name/ description }\end{array}$ & $\begin{array}{l}\text { Addgene ID for plasmid } \\
\text { containing part }\end{array}$ \\
\hline \multicolumn{5}{|c|}{ REGULATORY PARTS } \\
\hline PDC1pr/P2 & K. marxianus CBS6556 & 2/Promoter & Pyruvate decarboxylase/KMXK_OF05000 & 125035 \\
\hline ENO1pr/P3 & K. marxianus CBS6556 & 2/Promoter & Enolase/KMXK_OA03750 & 125036 \\
\hline TDH1pr/P4 & K. marxianus CBS6556 & 2/Promoter & $\begin{array}{l}\text { Glyceraldehyde-3-phosphate dehydrogenase } \\
\text { isozyme 1/KMXK_OD02420 }\end{array}$ & 125037 \\
\hline HSP150pr/P5 & K. marxianus CBS6556 & 2/Promoter & $\begin{array}{l}\text { Hypothetical cell wall manno-protein } \\
\text { HSP150/KMXK_OE02570 }\end{array}$ & 125038 \\
\hline INU1pr/P6 & K. marxianus CBS6556 & 2/Promoter & Inulinase/KMXK_0A03230 & 125039 \\
\hline TEF1pr/P7 & K. marxianus CBS6556 & 2/Promoter & $\begin{array}{l}\text { Translation elongation factor EF } \\
\text { alpha-1/KMXK_0G03180 }\end{array}$ & 125040 \\
\hline REV1pr/P8 & K. marxianus CBS6556 & 2/Promoter & Deoxycytidyl transferase/KMXK_OF03560 & 125041 \\
\hline HSP104pr/P13 & K. marxianus CBS6556 & 2/Promoter & Heat shock protein 104/KMXK_OD01210 & 125046 \\
\hline SSA2pr/P14 & K. marxianus CBS6556 & 2/Promoter & Heat shock protein SSA2/KMXK_OB04700 & 125047 \\
\hline TDH3pr/P15 & K. marxianus NBRC1777 & 2/Promoter & $\begin{array}{l}\text { Glyceraldehyde-3-phosphate dehydrogenase } \\
\text { isoform 3/KMAR_80062 }\end{array}$ & 125048 \\
\hline FBA1pr/P16 & K. marxianus CBS6556 & 2/Promoter & $\begin{array}{l}\text { Fructose 1,6-bisphosphate } \\
\text { aldolase/KMXK_OD04110 }\end{array}$ & 125049 \\
\hline XYL1pr/P17 & K. marxianus CBS6556 & 2/Promoter & Xylose reductase/KMXK_OA01570 & 125050 \\
\hline XYL2pr/P18 & K. marxianus CBS6556 & 2/Promoter & Xylitol dehydrogenase/KMXK_OH0042O & 125051 \\
\hline LAC4pr/P19 & K. marxianus CBS397 & 2/Promoter & Beta-galactosidase $^{\mathrm{C}}$ & 125052 \\
\hline INU1t/T1 & K. marxianus CBS6556 & 4/Terminator & Inulinase & 125053 \\
\hline LAC4t/T2 & K. marxianus CBS6556 & 4/Terminator & Beta-galactosidase & 125054 \\
\hline KmARS/CEN5/C2 & K. marxianus CBS6556 & 7/Origin & ARS and centromere from chromosome $V$ & 125060 \\
\hline KMARS/CEN6/C3 & K. marxianus CBS6556 & 7/Origin & ARS and centromere from chromosome $\mathrm{VI}$ & 125061 \\
\hline ARS2/C4 & K. marxianus CBS6556 & 7/Origin & Minimal ARS from chromosome II & 125063 \\
\hline \multicolumn{5}{|c|}{ HOMOLOGY ARMS FOR INSERTION VECTORS } \\
\hline I1L/11R & K. marxianus CBS6556 & 1,7 & Chromosome I:10187..11936 (LAC4) & $125030 / 125063$ \\
\hline I2L/I2R & K. marxianus CBS6556 & 1,7 & $\begin{array}{l}\text { Chromosme V: } 23743 . .21744 \text { (Crick Strand) } \\
\text { (downstream of } \mathrm{KmBDH1} \text { and } \mathrm{KmBDH2} \text { ) }\end{array}$ & $125031 / 125064$ \\
\hline I3L//3R & K. marxianus CBS6556 & 1,7 & $\begin{array}{l}\text { Chromosome IV:388650..390345 (between } \\
\text { KmSWF1 and KmARO1) }\end{array}$ & $125032 / 125065$ \\
\hline 14L/I4R & K. marxianus CBS6556 & 1,7 & $\begin{array}{l}\text { Chromosome IV:240042..241741 (downstream of } \\
\text { KmHSP104) }\end{array}$ & 125033/125066 \\
\hline \multicolumn{5}{|l|}{ OTHER PARTS } \\
\hline SCTRP1/M1 & S. cerevisiae CEN.PK 113-7D & 6/Marker & $\begin{array}{l}\text { Phosphoribosyl anthranilate isomerase expression } \\
\text { cassette from S. cerevisiae }\end{array}$ & 125058 \\
\hline
\end{tabular}

${ }^{a}$ Based on the CBS6556 genome annotations generated in Varela et al. (2017); ${ }^{b}$ Based on the NBRC annotations in (Inokuma et al., 2015); ${ }^{c}$ Based on the genome sequence assembled in Ortiz-Merino et al. (2018).

A plasmid backbone was then amplified from the original pUDP002 using primers pUDP002-F and pUDP002-R, and Gibson assembly was used to create pUCC001 from the two parts.
When using CRISPR/Cas9 to edit a site in the genome, a gRNA sequence targeting the gene of interest was first predicted using the sgRNA software (Xie et al., 2014). Complementary 
oligonucleotides comprising the gRNA sequence are designed with $5^{\prime}$ and $3^{\prime}$ overhangs ( $5^{\prime}$-CGTC- $3^{\prime}$ and $5^{\prime}-\mathrm{AAAC}-3^{\prime}$,) on the sense and antisense oligonucleotides respectively, creating sticky ends to be ligated into pUCC001 cut by BsaI. One hundred pico mole oligos are phosphorylated with $1 \mu \mathrm{L}$ of T4 polynucleotide kinase $\left(10 \mathrm{U} \mu \mathrm{L}^{-1}, \mathrm{NEB}\right)$ in a total volume of $10 \mu \mathrm{L}$ and then denatured and annealed. Fifty femto mole of the annealed gRNA insert is then used with $100 \mathrm{ng}$ of pUCC001 in a Golden Gate reaction with BsaI. Following transformation, the correct insertion of the gRNA was subsequently verified by PCR using the primer Bsa-R and the sense oligo containing the gRNA target, and sequencing of the plasmid. gRNA targets used in this study are listed in Table S5.

Three hundred nanograms of a gRNA expression plasmid are used in a typical genome engineering experiment. As K. marxianus predominantly repairs double stranded breaks by non-homologous end-joining (Daley et al., 2005), mutations are created around the gRNA target site without providing a repair fragment (Cernak et al., 2018). After 48-72 h growth following a transformation, hygromycin-resistant colonies are then screened for mutations at the targeted locus by colony PCR and sequencing (Jakociunas et al., 2015). If the intended mutations create an observable phenotype (e.g., an auxotrophy), the transformed plate is replica-plated to appropriate medium to pre-screen colonies based on the phenotype. Colonies with frameshift mutations are then grown overnight in YPD and then passaged twice to fresh YPD cultures to ensure loss of the gRNA plasmid before being preserved. When multiple genes were to be edited, sequential mutation was performed; following each mutation, the gRNA plasmid was cured and the mutation confirmed before proceeding with transformation of the next gRNA expression plasmid.

\section{RESULTS}

\section{A Collection of Parts and Assembly Pipeline for Kluyveromyces marxianus Using the Yeast Toolkit Standard}

The biological parts collected and characterized here are intended to provide the same functions for $K$. marxianus in all the relevant categories of YTK parts (Figure 1A). A number of the parts have been described and identified elsewhere (Bergkamp et al., 1993; Yang et al., 2015a), but we also provide a more substantial number of characterised promoters, terminators and integration sites for expression cassettes. A number of highand low-copy number origins have also been identified from K. marxianus genomes, or created from minimal elements. We have included three centromeric elements-one minimal (Yarimizu et al., 2013) and two genomic (Iborra and Ball, 1994; Ball et al., 1999)—and a minimal ARS element (Cernak et al., 2018) for the construction of expression vectors (Table 2). While the parts can be used to construct $K$. marxianus expression systems in general, they are optimally compatible with selection markers, bacterial vectors and synthetic connectors in the original YTK available from Addgene (\#1000000061). They allow the in vitro construction of cloning and expression systems for $K$. marxianus with the same flexibility one can for $S$. cerevisiae. Golden Gate assembly is an established in vitro assembly technique, and with it we were able to assemble up to 8 part-containing plasmids into reporter plasmids to characterize our parts.

\section{A Set of Native Promoters to Fine-Tune Gene Expression}

When selecting native yeast promoters to use in strain engineering, an important source is gene expression studies under conditions of interest. While a number of gene expression studies have been carried out on $K$. marxianus strains, few of them have focused on strains with the potential to be synthetic biology chassis, or on have instead focused on explicitly industrial conditions (Gao et al., 2015; Schabort et al., 2016; Diniz et al., 2017). To select promoters, we turned to two studies based on the strain DMKU3-1042, which also has the best quality publicly-available genome. One was TSS-seq transcriptome data published alongside its genome (Lertwattanasakul et al., 2015), as well as a smallscale gene expression study using fluorescent reporters of genes involved in carbon metabolism (Suzuki et al., 2015). The former gave us the opportunity to select not only promoters with distinct strengths, but also those induced by high temperature and xylose. Following this, the corresponding promoters were then identified from the CBS6556 genome. We excluded promoters with internal BsaI or BsmBI sites, as we lacked information on regulatory elements to justify removing the sites by mutagenesis.

This analysis allowed us to select 19 promoters: 10 constitutive and 9 inducible by heat, xylose, lactose and inulin, whose strengths we characterised using YFP reporter assays (Table 2, Figure 2A; Figure S1). While the promoter sequences came from CBS6556, we tested them in strain NBRC1777, due to its faster growth rate and superior thermotolerance compared to the former. Under standard conditions $\left(30^{\circ} \mathrm{C}\right.$, glucose-rich medium), we provide a broad selection of promoter strengths for gene expression. From the weakest (REV1pr) to the strongest (PDC1pr) promoters, we can achieve a 40-fold range of gene expression in NBRC1777. The strongest promoters were those of genes involved in central carbon metabolism (PDC1pr, FBA1pr, TDH3pr), as well as that of the orthologue of the translation elongation factor EF-1 $\alpha$ (TEF1) in S. cerevisiae. Interestingly, several of the same orthologous genes in S. cerevisiae also have strong promoters. However, the latter do not always achieve strong expression when used in K. marxianus (Figure S2) a trade-off against the advantages of using an orthogonal yeast promoter.

Kluyvermomyces marxianus's thermotolerance provides a unique induction signal for this yeast's promoters. With this in mind, we picked three heat-inducible promoters (HSP104pr, SSA2pr, TSA1pr) with different fold-induction at high temperatures, and one intended to be stable at ambient and high temperatures (HSP150pr) based on expression data for the strain DMKU3-1042 as earlier (Lertwattanasakul et al., 2015). All of our inducible promoters selected from expression data 


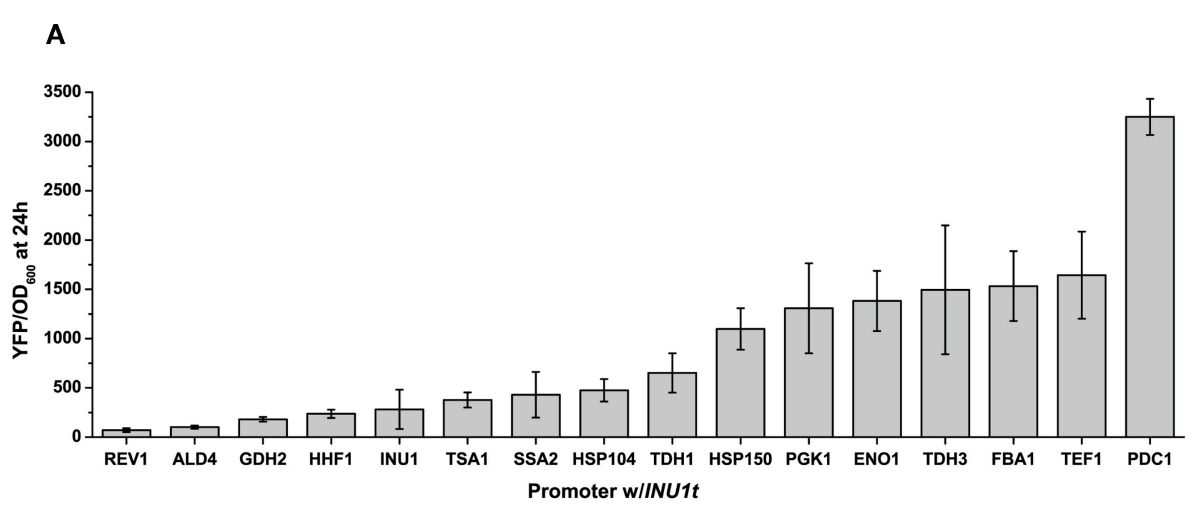

B

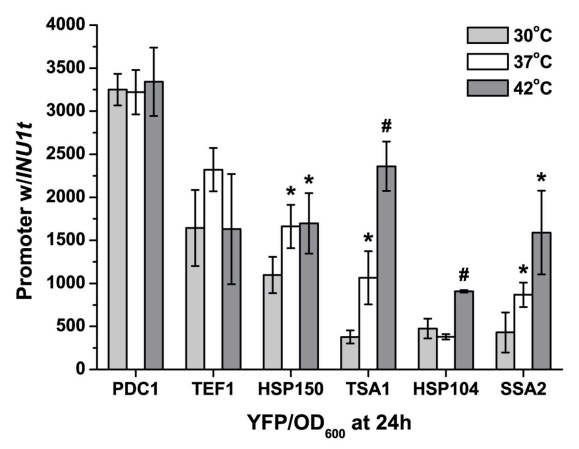

C

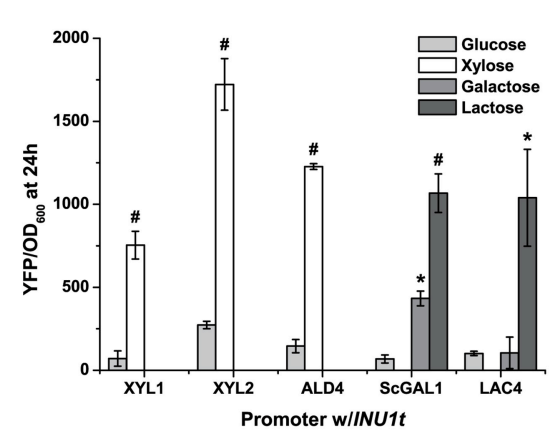

FIGURE 2 | Characterization of $K$. marxianus promoters. (A) A set of constitutive promoters, taken from genes with diverse functions, provide a wide range of expression levels - nearly two orders of magnitude-as seen from the YFP output under standard conditions $\left(24 \mathrm{~h}\right.$ at $30^{\circ} \mathrm{C}, 2 \%$ glucose in synthetic complete medium) (B) Expression of heat-inducible promoters at three different temperatures at which NBRC1777 can grow. Constitutive promoters as well (PDC1pr) have a stable output at high temperatures. (C) The diverse carbon source utilization of $K$. marxianus gives us a unique induction signal for this yeast, as seen by the induction of promoters by lactose, galactose, and xylose. All data are normalised to cell number using OD, and are plotted as the mean \pm s.d. of at least three replicates. YFP values significantly different from those under baseline conditions (30 degrees in drop-out medium with $2 \%$ glucose) are marked with an asterisk $(p<0.05)$ or a hash $(p<0.001)$.

exhibited induction under the relevant conditions, though not all to the same extent (Table S6). When tested at 37 and $42^{\circ} \mathrm{C}$, TSA1pr, SSA2pr, and HSP104pr promoters induced to different levels. Expression of YFP by HSP150pr, as predicted, remained relatively stable at higher temperatures, inducing weakly when exposed to $37^{\circ} \mathrm{C}$ but no further at $42^{\circ} \mathrm{C}$ (Figure 2B). The other three promoters offer a range of induction varying from 2 to 6.5-fold depending on the temperature. TSA1pr has the strongest fold-induction at $42^{\circ} \mathrm{C}$ and the highest measured fluorescence at high-temperature, whereas that of SSA2pr remains at increasing medium-to-high levels (relative to the constitutive promoters) as temperature increases (Figure 2B). In comparison, two strong promoters at $30^{\circ} \mathrm{C}-P D C 1 p r$ and TEF1pr-had their YFP expression relatively unchanged by increased temperature.

Another feature of $K$. marxianus's physiology that makes it an attractive cell factory chassis is its ability to utilize a wider range of carbon sources than $S$. cerevisiae, thus circumventing the need to engineer this yeast to consume the carbon sources in question. Wild-type K. marxianus can consume sugars found in plant (xylose and inulin) and dairy (lactose) waste, thus allowing reduced costs if using these as feedstocks for fermentations. For xylose induction, we cloned and tested the induction of three xylose-inducible promoters: XYL1pr, $X Y L 2 p r$, and ALD4pr. The xylose reductase XYL1 reduces xylose to xylitol, this in turn converted to xylulose by the xylitol dehydrogenase XYL2. XKS1 then phosphorylates it to xylulose5-phosphate, which can then enter the non-oxidative branch of the pentose phosphate pathway. ALD4pr was chosen from a genome-wide expression study for its low background in glucose and high fold-induction by xylose (Lertwattanasakul et al., 2015). These promoters exhibited a 6-to-10-fold increase in YFP using xylose as a carbon source relative to glucose, with XYL2pr having the strongest fold-induction (Figure 2C). For a lactose-inducible promoter, we chose the promoter for the beta-galactosidase LAC4. Unlike the other promoters in this set, we chose LAC4pr from strain CBS397, known to grow well on lactose as a carbon source (Varela et al., 2017). It exhibited a 10-fold induction by lactose relative to glucose alone when used to express YFP in NBRC1777. In comparison, the GAL1 promoter from $S$. cerevisiae was inducible by both galactose and lactose, but more strongly for the former (15-fold vs. 6fold, Figure 2C). 


\section{Terminators Provide a Second Level of Control Over Gene Expression}

Besides promoters, terminators can also control gene expression by affecting the lifetime of mRNA, and provide us with an extra means to fine-tune gene expression (Curran et al., 2013). While the terminators included with the original YTK aimed to keep gene expression output roughly constant (Lee et al., 2015), we have provided five native terminators to broaden the range of gene expression our parts can achieve (Figure 3A). Interestingly, two terminators from the YTK-those for ScADH1 and $S c P G K 1$ - can change gene expression between them (as measured by YFP fluorescence) by nearly a factor of two in $K$. marxianus. The full ability of promoters to further optimise gene expression can be seen when a set of four terminators and three terminators are combinatorically used to express YFP. While the promoter of choice is still the dominant factor in determining the level of gene expression, choosing a "weak" or "strong" terminator can significantly affect expression as well (TSA1pr vs. TDH1pr, Figure 3B). In the case of inducible promoters, terminator effects are more pronounced under non-inducible conditions. Nonetheless, the increase in basal expression as seen in the inulinase promoter due to a change in a "stronger" terminator is enough to halve fold-induction by inulin (Figure 3C). In summary, depending on the promoter and the means of its induction, the terminators we provide could be used to minimise background, maintain a level of basal expression, or smooth out changes in expression during different conditions if needed when expressing a heterologous gene.

\section{Efficient CRISPR/Cas9 Editing With pUCC001}

Our CRISPR/Cas9 editing plasmid pUCC001 takes advantage of the cross-species pUDP002 system and makes it a more flexible and economical tool by introducing a BsaI cloning site for new gRNA targets (Figure 4A). In this way, annealed oligos containing overhangs matching those generated by the BsaI sites in pUCC001 can be easily cloned into a cut plasmid, or using Golden Gate assembly. As a proof of principle, we transformed $K$. marxianus with pUCC001 containing a gRNA targeting the LAC4 locus. lac4 mutants are unable to convert XGal to a blue dye. We observed that over $50 \%$ of the hygromycinresistant colonies did not turn blue when grown on medium containing galactose and X-Gal, as opposed to $\sim 10-20 \%$ when a deletion cassette is used without CRISPR/Cas9 (Figure 4B, Table 3). As a demonstration of more practical applications, we used pUCC001 to rapidly generate defined single, double and triple auxotrophs for uracil, leucine, and histidine (Figures 4C,D; Table 1). Using gRNA plasmids targeting the $K$. marxianus orthologues of URA3, HIS3, and LEU2 we created frameshift mutations in these genes leading to loss of function. Given that no repair fragment was used, the efficiency of mutations was good $(\geq 50 \%)$; in general, we were able to retrieve the frameshift mutants in the figure by screening fewer than 8 colonies per transformation. The auxotrophic mutants so created allow us to use the orthogonal metabolic markers from the original YTK (ScURA3, ScLEU2, and ScHIS3), and show insignificant background when used in transformations with metabolic markers. Separately, the pUCC001 system was used to construct mutants in several other $K$. marxianus genes (Varela et al., 2019).

\section{Inactivating Different Genes Involved in Non-homologous End-Joining Has Different Effects on Gene Targeting Efficiency}

While metabolically engineering strains it is advantageous to integrate heterologous gene cassettes for stronger and more stable expression. In yeasts other than S. cerevisiae, the ability to efficiently target gene integration is hindered by their usage of non-homologous end-joining (NHEJ) as the dominant DNA repair mechanism (Daley et al., 2005). As a result, random and incomplete integrations are frequent, and much larger targeting homology sequences are also required (up to $1 \mathrm{~kb}$ ) compared to $S$. cerevisiae (50 bp) for a successful integration (Baudin et al., 1993; Choo et al., 2014). While random integrations of multiple copies of a gene can be advantageous in some biotechnology applications (Lin et al., 2017), it is equally important to integrate single copies of heterologous genes or pathways to rationally construct a cell factory or evaluate different metabolic engineering strategies. Inactivating any of the key genes involved in NHEJ-YKU70/80, NEJ1 or DNL4 (Abdel-Banat et al., 2010; Choo et al., 2014; Nambu-Nishida et al., 2017) — has been shown to increase targeted integration in $K$. marxianus by forcing it to use homologous recombination (HR) alone for DNA repair. It remains unclear if the different mutations suppress random integration to the same extent. To decide which NHEJ mutant gave us the highest rate of targeted integration, we tested the integration of a YFP expression cassette at the LAC4 locus in backgrounds that were either wild-type or had either YKU80, DNL4, or NEJ1 previously inactivated by CRISPR/Cas9 (YBL001, KmASR.005, and YBL003, respectively; Table 1). The cassette was flanked by 880 bp on either end targeting LAC4 (Figure 5A). As with inactivation by CRISPR/Cas9, correct integration would disrupt the gene and render the yeast unable to break down $\mathrm{X}$ Gal, allowing us to pre-screen colonies by blue/white selection for genotyping (Figure 4B). The YFP cassette would also allow us to determine if transformants contained multiple integrants in a semi-quantitative manner. Sequencing around the insertion site revealed that integration of the cassette was "seamless" in all backgrounds; the sequencing region immediately downstream of the insertion sites revealed no mutations surrounding the insertion point (Figure 5A).

Our experiments revealed that inactivating NHEJ resulted in disruption of the LAC4 locus in nearly all transformants, as opposed to only occurring for about $\sim 10 \%$ of transformants in wild-type $K$. marxianus. Furthermore, all white colonies of the NHEJ-deficient strains had the YFP cassette correctly integrated, as opposed to $<20 \%$ for the wild-type strain (Table 3 ). In spite of correct integration, wild-type transformants still had a wide spread of measured fluorescent intensities (Figure 5B). This 
A

B
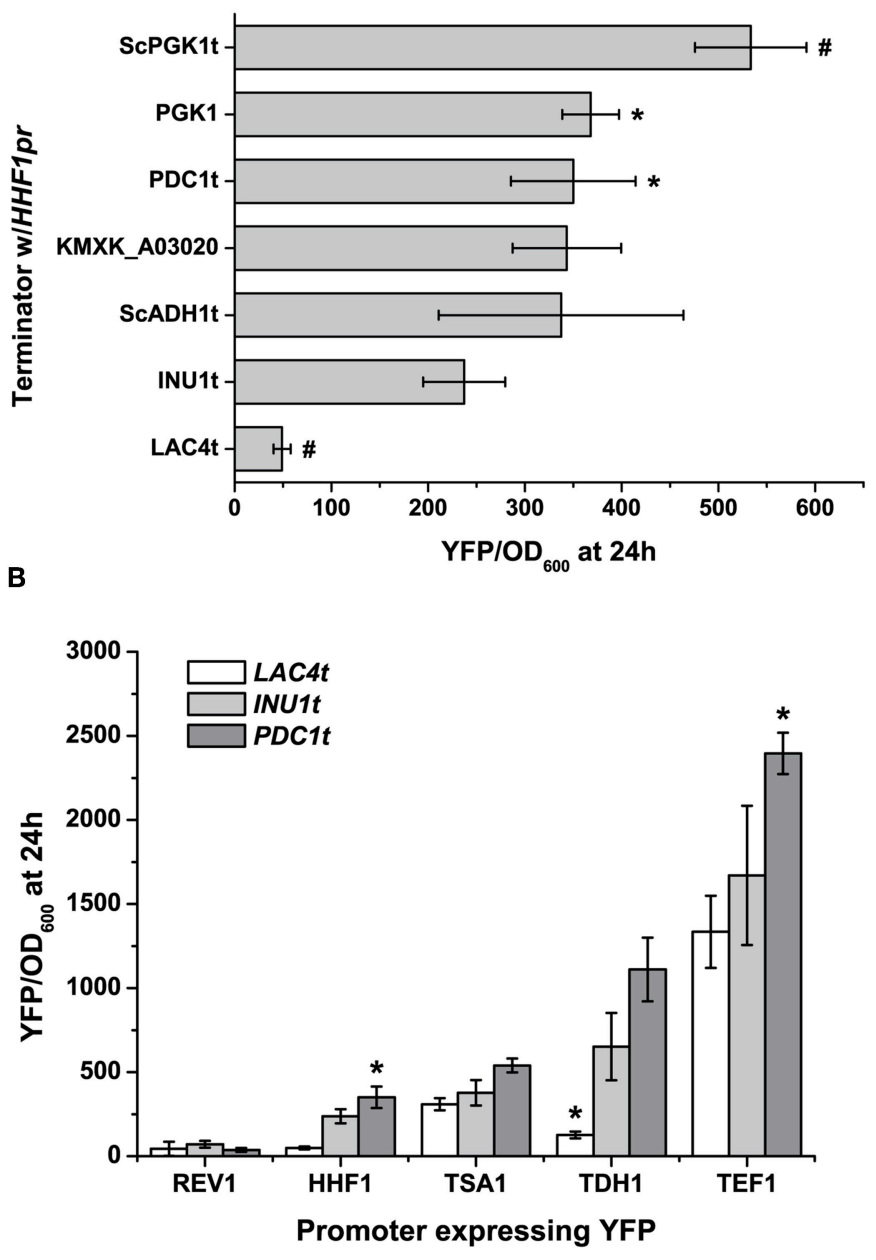

C

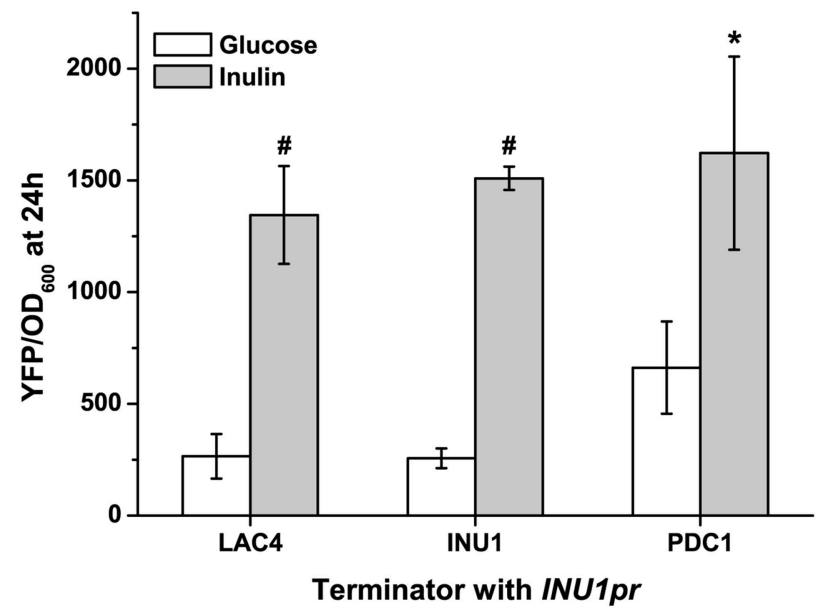

FIGURE 3 | Using terminators to fine-tune gene expression. (A) Terminator choice can change gene expression by a factor of nearly 5 using native terminators, and this range can be even further exchanged if terminators from S. cerevisiae are used. (B) This can further be used to control gene expression which promoters and terminators are used combinatorially, though the starting strength of the promoter can influence the range. (C) Inducible promoters' expression is largely altered under non-inducing conditions when used to express YFP with different terminators. Here the strong induction of the inulinase (INU1) promoter by inulin is not significantly affected by terminator choice, while it can affect leaky expression under non-inducing conditions. All data are plotted as the mean $\pm \mathrm{s}$.d. of at least three replicates. YFP values significantly different from those under baseline conditions (expression using INU1t) are marked with an asterisk $(p<0.05)$ or a hash $(p<0.001$ ). 

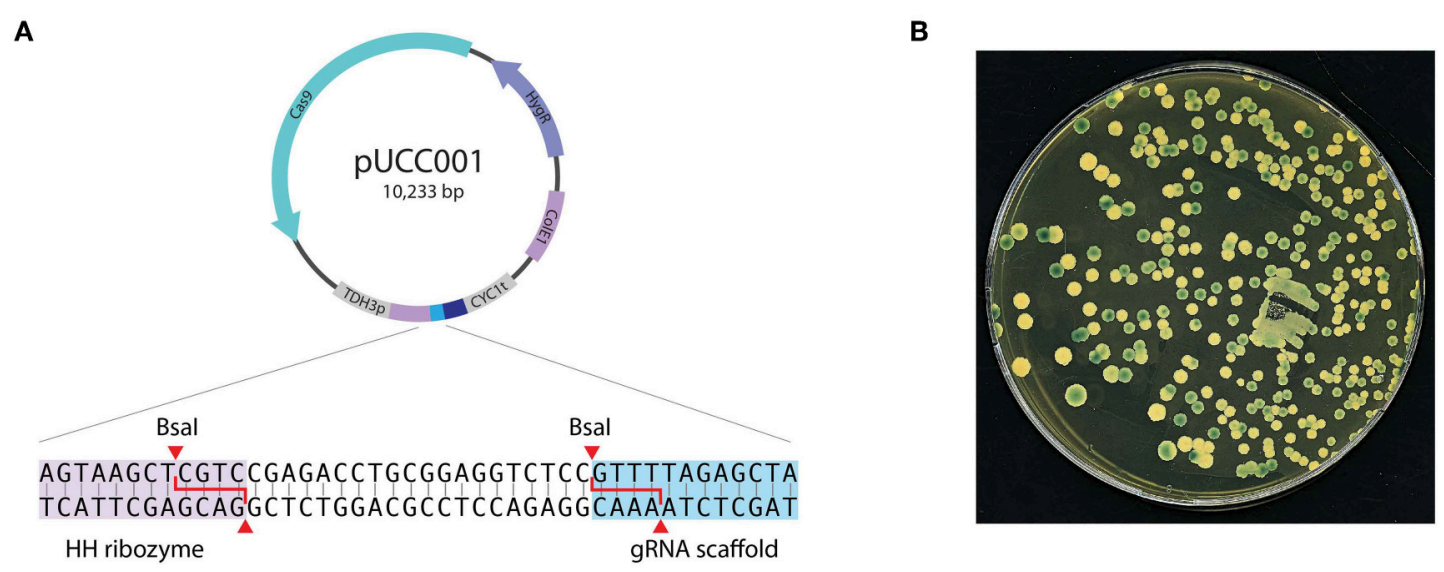

C
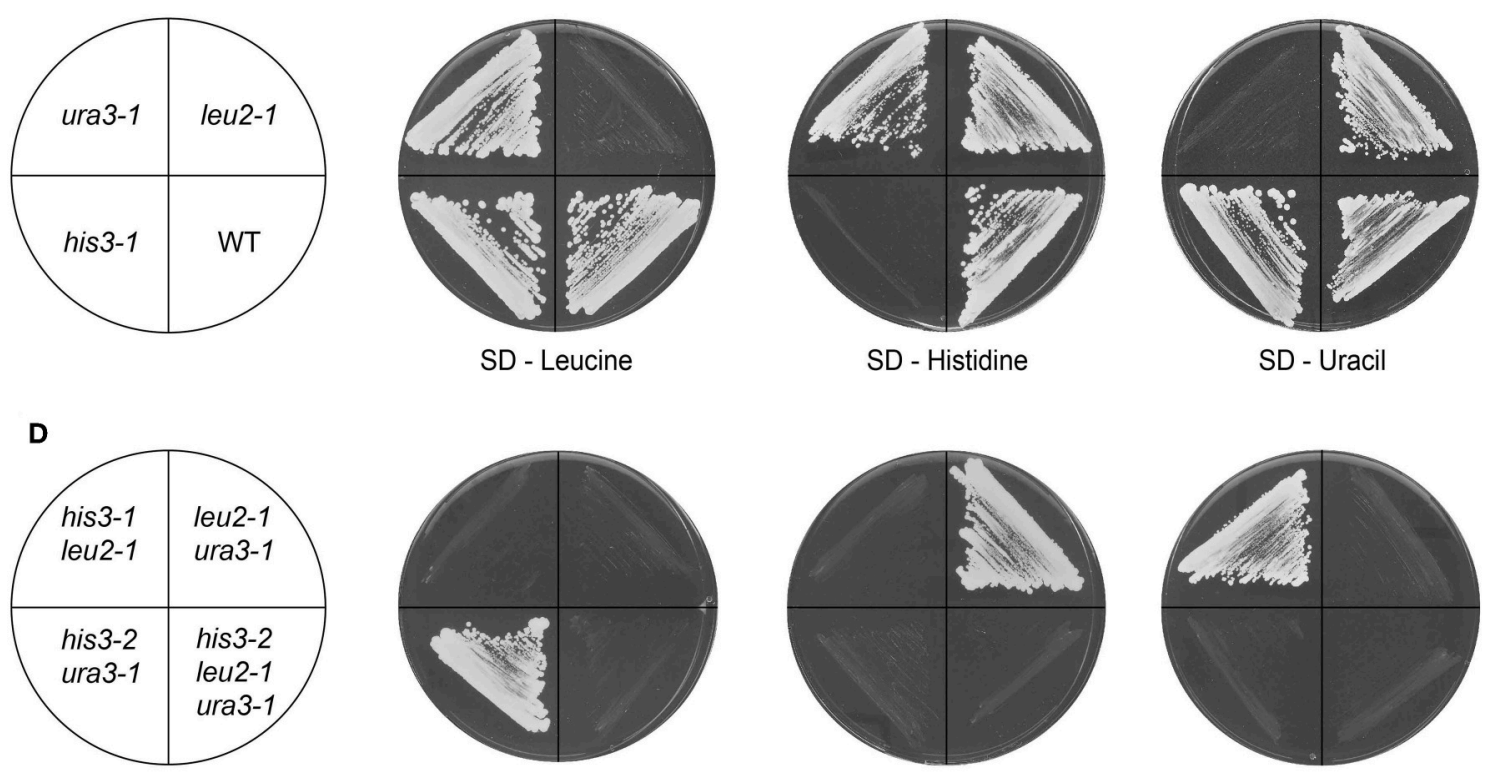

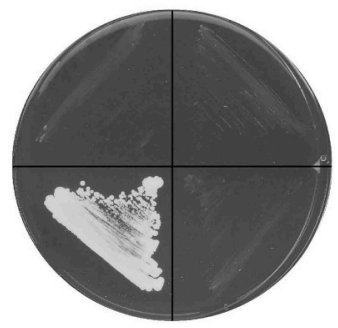

SD - Leucine

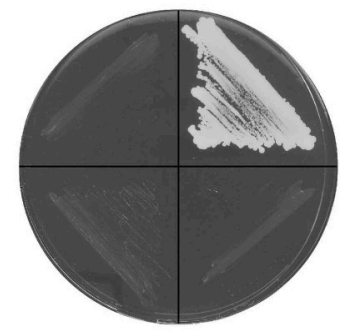

SD - Histidine

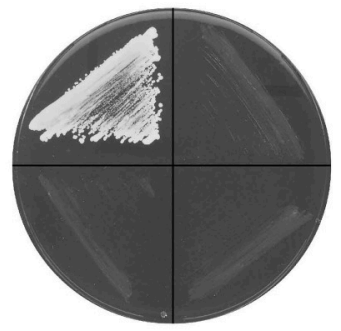

SD - Uracil

FIGURE 4 | CRISPR/Cas9 genome editing with pUCC001. (A) Map of the plasmid. Based on pUDP002, the plasmid contains Cas9 expressed using the A.adeninivorans promoter, a pangenomic ARS and a gRNA expression cassette under the control of the S. cerevisiae TDH3 promoter. The inset shows the cloning site inserted between the ribozyme units; two Bsal sites allow the construction of new gRNA targets using Golden Gate cloning. (B) Proof-of-principle of pUCC001 function by inactivation of the LAC4 locus. Colonies grown YPGal + X-Gal, with a functioning copy of LAC4 should produce a blue colour on cleaving X-Gal; here, over half of the colonies remain white due to CRISPR-mediated inactivation of LAC4. (C,D) Construction of single and multiple auxotrophs. With pUCC001, we were able to rapidly construct genome editing plasmids targeting orthologues of genes commonly inactivated in S. cerevisiae laboratory strains: URA3, LEU2 and HIS3, both as (C) single and (D) multiple mutants. Frameshift mutations in each gene created the desired auxotrophies, and the strains so generated could be targeted with other plasmids to create defined double and triple mutants.

led us to hypothesise that correct integration in a wild-type strain does not preclude random integration. The contrasting low variation in YFP fluorescence seen in DNL4 and YKU80 mutants suggests that these backgrounds incorporate a single copy of the YFP cassette. On the other hand, NEJ1 mutants seemed to incorporate multiple copies of YFP. In the case of the latter, it is admittedly unclear if these extra copies are integrated sequentially at LAC4, or elsewhere in the genome. In summary, it is clear that DNL4 or YKU80 are the best NHEJ components to inactivate to ensure targeted, single chromosomal integrations of DNA. Extrapolating from research in S. cerevisiae, our findings are given weight by the different roles in NHEJ of the proteins we targeted. YKU80, as part of the Ku complex, is the first protein to bind to and stabilise double-stranded breaks, followed by DNL4 (Emerson and Bertuch, 2016). Therefore, the role of these "first responders" in DNA repair might make them better targets to thoroughly inactivate NHEJ. Furthermore, as other research has found that the binding of DNL4 to DNA may not require NEJ1 
TABLE 3 | Gene targeting and integration efficiencies at the LAC4 locus in different backgrounds.

\begin{tabular}{lcccc}
\hline Background/strain & G418-resistant colonies & White resistant colonies & Gene disruption efficiency, \% ${ }^{\mathbf{a}}$ & Gene targeting efficiency, \% $^{\mathbf{b}}$ \\
\hline Wild-type/NBRC1777 & $863 \pm 36$ & $111 \pm 13$ & $12.9 \pm 1.3$ & $16.7 \pm 5.9$ \\
yku80-1/YBL001 & $587 \pm 71$ & $587 \pm 71$ & 100 & 100 \\
dnl4-1/KmASR.005 & $456 \pm 44$ & $455 \pm 44$ & 99.93 & 100 \\
nej1-1/YBL003 & $469 \pm 32$ & $466 \pm 32$ & 99.36 & 100 \\
\hline
\end{tabular}

The data are presented as the mean \pm s.d of three replicates.

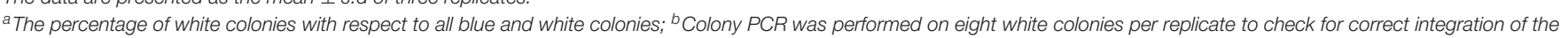
YFP cassette at LAC4.

A

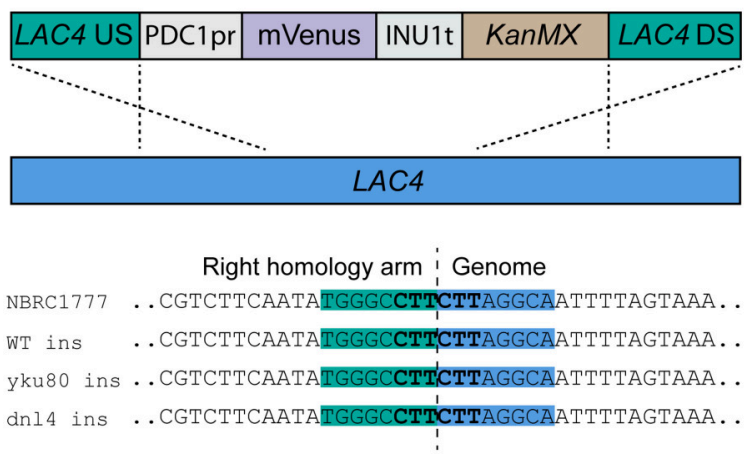

C

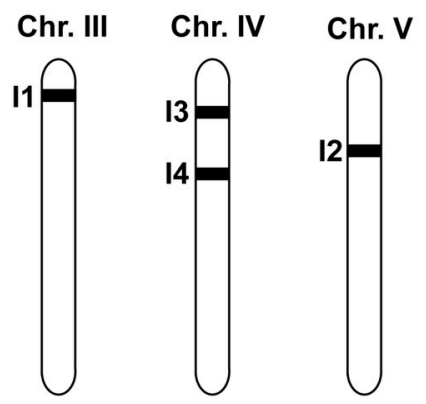

B

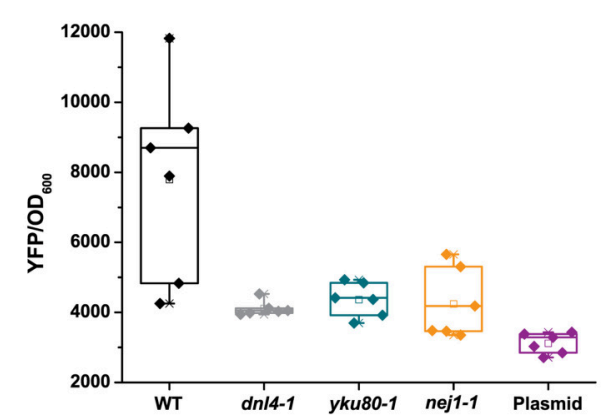

D

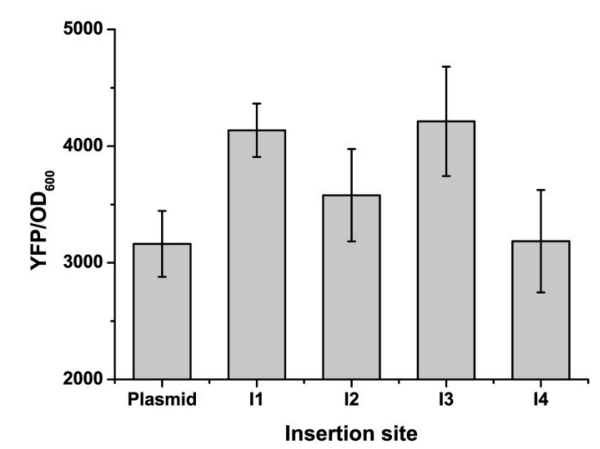

FIGURE 5 | Random and targeted integration in K. marxianus. (A) Using homology arms 880 bp in length, integration of a YFP reporter cassette (with marker) is seamless, with no sequence loss or modifications at the integration site irrespective of the genetic background (inset). (B) Inactivating YKU80 or DNL4 but not NEJ1 is essential to eliminate random integrations. When targeting an YFP expression cassette at the LAC4 locus, correct integration in a wild-type strain (as determined by genotyping) does not abolish random integration elsewhere, as seen by the spread in YFP fluorescence measured from five transformed colonies. YFP expression for the same cassette expressed on a centromeric plasmid is provided as a reference. (C) Genomic locations of insertion sites for gene expression cassettes, selected as described in the main text. (D) The effect of genetic context on expression levels of the same gene. The bar plots are plotted as the mean \pm s.d. of at least three replicates.

(Wu et al., 2008), and the latter enhances but is not essential for NHEJ entirely (Yang et al., 2015b), this may explain why the inactivation of NEJ1 in YBL003 was insufficient to ensure single-copy gene integration. In general, expression for a single integrated cassette was higher than that when the same cassette was expressed on a low-copy-number plasmid using the same antibiotic marker (Figure 5B); the same trend was seen across several promoters (Figure S3).

\section{Evaluation of Sites for Chromosomal Integration}

While selecting chromosomal integration sites in the K. marxianus genome, we considered three criteria: (i) providing a visible or easily detectable phenotype on integration, simplifying screening if necessary, (ii) near clusters of activelytranscribed genes, and (iii) near essential genes to ensure that only cells with correctly-inserted DNA would survive(Mikkelsen 
et al., 2012). For insertion sites of the first type, we chose the LAC4 locus (I1); insertion there has a visible phenotype that does not affect core metabolism (as opposed to $A D E 2$, for example). For selecting sites of the second type, we examined existing TSS-seq data (Lertwattanasakul et al., 2015) and found two such sites where the gene clusters were on the same coding strand: one each on chromosome IV and V (I2 and I4) (Figure 5C; Table 2). We also selected an insertion site upstream of $A R O 1$, the pentafunctional protein involved in aromatic amino acid biosynthesis (I3); an incorrect insertion would result in auxotrophy for the aromatic amino acids phenylalanine, tyrosine, and tryptophan. While cloning the 5' (left) homology arms into level I plasmids, we added the overhangs matching the 5' multigene connector ConLS' by PCR, turning it into a type 1 part. This included the nested connector sequence containing the BsmBI site with the appropriate overhang for multigene constructs, which we added by PCR. This way the part with the homology arm can also be used to construct integrative vectors for a single expression cassette, as it still possesses the appropriate BsaI overhang at its 3' end to clone a TU in, as described below. The 3' (right) homology arms, as in the original YTK, contain BsaI overhangs corresponding to a yeast replicative origin, or type 7 part (Lee et al., 2015). Finally, the homology arms have AscI restriction sites directly flanking them to linearise the insertion vector prior to transformation, to improve integration efficiency.

Using these arms in place of connectors provided for TUs in the original YTK, we constructed novel integrative reporter vectors, each expressing YFP under the control of the strongest promoter we identified (PDC1pr; P2) and targeting I1 to I4. We then evaluated the integration efficiency at each site in KmASR.005, in each case using the same YFP expression cassette. Insertion efficiency was $100 \%$ at all loci, and while variations in YFP expression were observed at all loci, none varied by more than 25\% (Figure 5D). However, this difference in expression may be more significant when expressing multiple genes in a heterologous biosynthetic pathway as opposed to a single fluorescent protein. Integrating the gene at I3, upstream of $A R O 1$, did not cause the auxotrophies for Phe, Trp, and Tyr expected if the gene was disrupted, suggesting that the integration there was seamless as well. However, insertion sites near essential genes may not always be this accessible; another site we tested near an orthologue of the translation initiation factor TIF1 yielded $<10 \%$ correct integrations in the wild-type strain and no colonies in an NHEJ-deficient background (data not shown).

\section{DISCUSSION}

Standardised biological parts for the expression and maintenance of genes, and means to assemble them, are a cornerstone of synthetic biology to build new biological systems. On a more practical level, they allow us to accelerate the design-build-test cycle which forms the core of applied synthetic biology and its sister discipline, metabolic engineering. A versatile collection of these parts is the Yeast Toolkit collected 3 years ago. Grouped into eight categories of parts, the standard set forth by the
YTK allows the hierarchical assembly of expression or storage vectors for expressing recombinant genes in S. cerevisiae with a wide range of selection markers, regulatory elements, and functional protein tags to fine-tune gene expression and engineer the genome as needed. Clearly, to move beyond being niche organisms in biotechnology, alternative yeasts require such part collections to make rapid metabolic engineering feasible. It is also advantageous to maintain a common standard for assembly to facilitate the exchange of parts between researchers and yeasts as needed. It was with this goal in mind that we selected and characterized the parts presented in this kit, while maintaining the YTK standard. Large part collections have been developed for other yeasts (Celinska et al., 2017; Prielhofer et al., 2017), and it is with such collections in mind that we have created ours. Using the established Golden Gate assembly protocols, we were able to assemble episomal and integrative reporter constructs from up to 8 component plasmids to characterize our parts.

Our extension of the YTK also includes the first collection of homology arms for insertion vectors targeting four loci in the $K$. marxianus genome, each with different characteristics. They are considered "full-length," but can easily be shortened using PCR or re-cloning in the case of their use in NHEJdeficient strains to an optimal length to minimize construct size without compromising gene targeting efficiency. As better genomic and transcriptomic knowledge of $K$. marxianus is acquired, more insertion sites and parts will be identified to be added to the modest set we provide. We also foresee the set being expanded by synthetic promoters, engineered promoters (as has been done with INU1pr) and secretion tags (Zhou et al., 2018).

While the promoters we have characterized are largely selected from strain CBS6556, all but one of them had $>90 \%$ sequence similarity with strain DMKU3-1042 and with strain NBRC1777, where the characterization was carried out. Within the observed differences, only a few promoters do exhibit significant sequence differences that could affect gene expression between strains based on orthologous transcription factor binding sites from S. cerevisiae (Table S7). Sequence differences between promoters for the same gene in different yeast strains can have implications in gene expression, and therefore should be taken into consideration for experimental and industrial applications (Liu et al., 2015; de Paiva et al., 2018); however, at this stage not enough is known about $K$. marxianus' native transcription factors and regulatory network to functionally dissect our promoter sequences. Their measured activity under the selected expression conditions, and in two different strains, demonstrate their practical usability.

Alongside parts collections, the existence of genome editing tools for allelic replacement and deletion speeds up the creation of strains with defined mutant genotypes and mating, similar to standard $S$. cerevisiae lab strains. This also opens up the possibility of creating the best $K$. marxianus strains for the laboratory and industry using classical genetics methods and synthetic biology side-by-side (Cernak et al., 2018; Lee et al., 2018). It is with this end in mind that our collection also provides pUCC001, a Cas9/gRNA genome editing plasmid derived from the broad-host platform pUDP002 (Juergens et al., 2018), into 
which gRNA targets can be rapidly cloned by Golden Gate assembly simply as annealed and phosphorylated oligos. This saves the cost and time of cloning the entire gRNA expression cassette for every target as for the original plasmid. We have also cloned the ScTRP1 expression cassette for use in future strains auxotrophic for tryptophan. The YTK standard makes it possible to multiplex gRNA expression using pUCC001. In theory, Cas9 and multiple gRNA cassettes could be separately cloned as level II plasmids and then reassembled into a level III "multi-TU" plasmid. The ease of assembly of both individual gRNA plasmids and the Golden Gate assembly would provide a credible, if not more efficient, alternative to existing multiplexing systems, (Löbs et al., 2018). However, further optimization of the gRNA expression system is necessary for an optimal K. marxianusspecific multiplexing system.

While investigating different NHEJ-deficient backgrounds, we found that inactivating YKU80 or DNL4, but not NEJ1, was the best way to eliminate multiple or random integration. Identifying such a background is beneficial to improve the efficiency and specificity of $K$. marxianus-based in vivo assembly techniques such as PGASO (Chang et al., 2012), and to further define a genotype for a potential future "lab strain" for K. marxianus. As much as the versatility of the YTK standard is of relevance to synthetic biologists and metabolic engineers, the parts we have gathered may be of broader interest in the long term. Kluyveromyces marxianus is slowly emerging from its niche applications to become an alternative cell factory to $S$. cerevisiae. Several efforts have been, and are being made, to make it produce bio-based compounds of value (Cheon et al., 2014; Kim et al., 2014; Lin et al., 2017). The lack of standardised parts, and efficient synthetic biology tools and strategies has limited the scope or sophistication of these efforts. We believe this collection can enrich the existing synthetic biology landscape of $K$. marxianus and allow researchers to make more informed choices for the

\section{REFERENCES}

Abdel-Banat, B. M. A., Nonklang, S., Hoshida, H., and Akada, R. (2010). Random and targeted gene integrations through the control of nonhomologous end joining in the yeast Kluyveromyces marxianus. Yeast 27, 29-39. doi: 10.1002/yea.1729

Awan, A. R., Blount, B. A., Bell, D. J., Shaw, W. M., Ho, J. C. H., McKiernan, R. M., et al. (2017). Biosynthesis of the antibiotic nonribosomal peptide penicillin in baker's yeast. Nat. Commun. 8, 1-8. doi: 10.1038/ncomms15202

Ball, M. M., Raynala, A., Guérineau, M., and Iborra, F. (1999). Construction of efficient centromeric, multicopy and expression vectors for the yeast Kluyveromyces marxianus using homologous elements and the promoter of a purine-cytosine-like permease. J. Mol. Microbiol. Biotechnol. 1, 347-353.

Baudin, A., Ozier-Kalogeropoulus, O., Denouel, A., Lacroute, F., and Cullin, C. (1993). A simple and efficient method for direct gene deletion in Saccharomyces cerevisiae. Nucleic Acids Res. 21, 3329-3330. doi: 10.1093/nar/ 21.14.3329

Bergkamp, R. J. M., Bootsman, T. C., Toschka, H. Y., Mooren, A. T. A., Kox, L., Verbakel, J. M. A., et al. (1993). Applied microbiology biotechnology expression of an a-galactosidase gene under control of the homologous inulinase promoter in Kluyveromyces marxianus. Appl. Microbiol. 40, 309-317. doi: 10.1007/BF00170386

Celinska, E., Ledesma-Amaro, R., Larroude, M., Rossignol, T., Pauthenier, C., and Nicaud, J. M. (2017). Golden Gate Assembly system dedicated to complex more efficient, predictable and practical design and testing of future cell factories for a bio-based economy.

\section{AUTHOR CONTRIBUTIONS}

AR and JV carried out the experimental work, interpreted the data and wrote the manuscript. HJ carried out the experimental work. J-MD and JM conceived the study, supervised the research, interpreted the data and contributed to writing the manuscript.

\section{FUNDING}

AR, JV, J-MD, and JM were supported by the CHASSY project which received funding from the European Union's Horizon 2020 research and innovation programme under grant agreement No. 720824. JV was a fellow in the YEASTCELL training network, which received funding from the People Programme (Marie Curie Actions) of the European Union's Seventh Framework Programme FP7/2007-2013/ under REA grant agreement no 606795. HJ was supported by the BE-Basic R\&D Program, which was granted an FES subsidy from the Dutch Ministry of Economic Affairs, Agriculture and Innovation (EL\&I).

\section{ACKNOWLEDGMENTS}

We thank Jasmijn Hassing and Macarena Larroudé for advice and discussions regarding parts assembly. We acknowledge the help of Amy Bergin and Beth Mulcahy in cloning the parts plasmids.

\section{SUPPLEMENTARY MATERIAL}

The Supplementary Material for this article can be found online at: https://www.frontiersin.org/articles/10.3389/fbioe. 2019.00097/full\#supplementary-material pathway manipulation in Yarrowia lipolytica. Microb. Biotechnol. 10, 450-455. doi: 10.1111/1751-7915.12605

Cernak, R., Estrella, R., Poddar, S., Skerker, J. M., Cheng, Y.-F., Carlson, A. K., et al. (2018). Engineering Kluyveromyces marxianus as a robust synthetic biology platform host paul. MBio 9, 1-16. doi: 10.1128/mBio.01410-18

Chang, J. J., Ho, C. Y., Ho, F. J., Tsai, T. Y., Ke, H. M., Wang, C. H., et al. (2012). PGASO: a synthetic biology tool for engineering a cellulolytic yeast. Biotechnol. Biofuels 5, 1-12. doi: 10.1186/1754-6834-5-53

Chang, J. J., Ho, F. J., Ho, C. Y., Wu, Y. C., Hou, Y. H., Huang, C. C., et al. (2013). Assembling a cellulase cocktail and a cellodextrin transporter into a yeast host for CBP ethanol production. Biotechnol. Biofuels 6, 1-13. doi: 10.1186/1754-6834-6-19

Cheon, Y., Kim, J. S., Park, J. B., Heo, P., Lim, J. H., Jung, G. Y., et al. (2014). A biosynthetic pathway for hexanoic acid production in Kluyveromyces marxianus. J. Biotechnol. 182-183, 30-36. doi: 10.1016/j.jbiotec.2014. 04.010

Choo, J. H., Han, C., Kim, J. Y., and Kang, H. A. (2014). Deletion of a KU80 homolog enhances homologous recombination in the thermotolerant yeast Kluyveromyces marxianus. Biotechnol. Lett. 36, 2059-2067. doi: 10.1007/s10529-014-1576-4

Curran, K. A., Karim, A. S., Gupta, A., and Alper, H. S. (2013). Use of expressionenhancing terminators in Saccharomyces cerevisiae to increase mRNA half-life and improve gene expression control for metabolic engineering applications. Metab. Eng. 19, 88-97. doi: 10.1016/j.ymben.2013.07.001 
Daley, J. M., Palmbos, P. L., Wu, D., and Wilson, T. E. (2005). Nonhomologous end joining in yeast. Annu. Rev. Genet. 39, 431-451. doi: 10.1146/annurev.genet.39.073003.113340

de Paiva, D. P., Rocha, T. B., Rubini, M. R., Nicola, A. M., Reis, V. C. B., Torres, F. A. G., et al. (2018). A study on the use of strain-specific and homologous promoters for heterologous expression in industrial Saccharomyces cerevisiae strains. AMB Express 8, 1-11. doi: 10.1186/s13568-018-0613-4

Denby, C. M., Li, R. A., Vu, V. T., Costello, Z., Lin, W., Chan, L. J. G., et al. (2018). Industrial brewing yeast engineered for the production of primary flavor determinants in hopped beer. Nat. Commun. 9, 1-10. doi: 10.1038/s41467-018-03293-x

Diniz, R. H. S., Villada, J. C., Alvim, M. C. T., Vidigal, P. M. P., Vieira, N. M., Lamas-Maceiras, M., et al. (2017). Transcriptome analysis of the thermotolerant yeast Kluyveromyces marxianus CCT 7735 under ethanol stress. Appl. Microbiol. Biotechnol. 101, 6969-6980. doi: 10.1007/s00253-017-8432-0

Emerson, C. H., and Bertuch, A. A. (2016). Consider the workhorse: nonhomologous end-joining in budding yeast ${ }^{1}$. Biochem. Cell Biol. 94, 396-406. doi: 10.1139/bcb-2016-0001

Feng, J., Jester, B. W., Tinberg, C. E., Mandell, D. J., Antunes, M. S., Chari, R., et al. (2015). A general strategy to construct small molecule biosensors in eukaryotes. Elife 4, 1-23. doi: 10.7554/eLife.10606

Fonseca, G. G., Gombert, A. K., Heinzle, E., and Wittmann, C. (2007). Physiology of the yeast Kluyveromyces marxianus during batch and chemostat cultures with glucose as the sole carbon source. FEMS Yeast Res. 7, 422-435. doi: 10.1111/j.1567-1364.2006.00192.x

Gao, J., Yuan, W., Li, Y., Xiang, R., Hou, S., Zhong, S., et al. (2015). Transcriptional analysis of Kluyveromyces marxianus for ethanol production from inulin using consolidated bioprocessing technology. Biotechnol. Biofuels 8, 1-17. doi: 10.1186/s13068-015-0295-y

Gietz, R. D. (2014). Yeast transformation by the LiAc/SS carrier DNA/PEG method. Methods Mol. Biol. 1163, 33-44. doi: 10.1007/978-1-4939-0799-1_4

Gombert, A. K., Madeira, J. V., Cerdán, M.-E., and González-Siso, M.-I. (2016). Kluyveromyces marxianus as a host for heterologous protein synthesis. Appl. Microbiol. Biotechnol. 100, 6193-6208. doi: 10.1007/s00253-016-7645-y

Iborra, F., and Ball, M. M. (1994). Kluyveromyces marxianus small DNA fragments contain both autonomous replicative and centromeric elements that also function in Kluyveromyces lactis. Yeast 10, 1621-1629. doi: 10.1002/yea.320101211

Inokuma, K., Ishii, J., Hara, K. Y., Mochizuki, M., and Hasunuma, T. (2015). Complete genome sequence of Kluyveromyces marxianus NBRC1777, a nonconventional thermotolerant yeast. Genome Announc. 3, 2-3. doi: 10.1128/genomeA.00389-15

Jakociunas, T., Rajkumar, A. S., Zhang, J., Arsovska, D., Rodriguez, A., Jendresen, C. B., et al. (2015). CasEMBLR: Cas9-facilitated multiloci genomic integration of in vivo assembled DNA parts in Saccharomyces cerevisiae. ACS Synth. Biol. 4, 1126-1134. doi: 10.1021/acssynbio.5b00007

Jeong, H., Lee, D.-H., Kim, S. H., Kim, H.-J., Lee, K., Song, J. Y., et al. (2012). Genome sequence of the thermotolerant yeast Kluyveromyces marxianus var . marxianus KCTC 17555. Eukaryot. Cell 11, 1584-1585. doi: 10.1128/EC.00260-12

Juergens, H., Varela, J. A., Gorter de Vries, A. R., Perli, T., Gast, V. J. M., Gyurchev, N. Y., et al. (2018). Genome editing in Kluyveromyces and Ogataea yeasts using a broad-host-range Cas9/gRNA co-expression plasmid. FEMS Yeast Res. 18, 1-16. doi: 10.1093/femsyr/foy012

Kim, T. Y., Lee, S. W., and Oh, M. K. (2014). Biosynthesis of 2-phenylethanol from glucose with genetically engineered Kluyveromyces marxianus. Enzyme Microb. Technol. 61-62, 44-47. doi: 10.1016/j.enzmictec.2014.04.011

Lee, K. S., Kim, J. S., Heo, P., Yang, T. J., Sung, Y. J., Cheon, Y., et al. (2013). Characterization of Saccharomyces cerevisiae promoters for heterologous gene expression in Kluyveromyces marxianus. Appl. Microbiol. Biotechnol. 97, 2029-2041. doi: 10.1007/s00253-012-4306-7

Lee, M. E., DeLoache, W. C., Cervantes, B., and Dueber, J. E. (2015). A highly characterized yeast toolkit for modular, multipart assembly. ACS Synth. Biol. 4, 975-986. doi: 10.1021/sb500366v

Lee, M. H., Lin, J. J., Lin, Y. J., Chang, J. J., Ke, H. M., Fan, W. L., et al. (2018). Genome-wide prediction of CRISPR/Cas9 targets in Kluyveromyces marxianus and its application to obtain a stable haploid strain. Sci. Rep. 8, 1-10. doi: $10.1038 / \mathrm{s} 41598-018-25366-\mathrm{Z}$
Lertwattanasakul, N., Kosaka, T., Hosoyama, A., Suzuki, Y., Rodrussamee, N., Matsutani, M., et al. (2015). Genetic basis of the highly efficient yeast Kluyveromyces marxianus: complete genome sequence and transcriptome analyses. Biotech. Biofuels 8:47. doi: 10.1186/s13068-015-0227-X

Lin, Y. J., Chang, J. J., Lin, H. Y., Thia, C., Kao, Y. Y., Huang, C. C., et al. (2017). Metabolic engineering a yeast to produce astaxanthin. Bioresour. Technol. 245, 899-905. doi: 10.1016/j.biortech.2017.07.116

Liu, J., Martin-Yken, H., Bigey, F., Dequin, S., François, J. M., and Capp, J. P. (2015). Natural yeast promoter variants reveal epistasis in the generation of transcriptional-mediated noise and its potential benefit in stressful conditions. Genome Biol. Evol. 7, 969-984. doi: 10.1093/gbe/evv047

Löbs, A. K., Engel, R., Schwartz, C., Flores, A., and Wheeldon, I. (2017). CRISPR-Cas9-enabled genetic disruptions for understanding ethanol and ethyl acetate biosynthesis in Kluyveromyces marxianus. Biotechnol. Biofuels 10, 1-14. doi: 10.1186/s13068-017-0854-5

Löbs, A. K., Schwartz, C., Thorwall, S., and Wheeldon, I. (2018). Highly multiplexed CRISPRi repression of respiratory functions enhances mitochondrial localized ethyl acetate biosynthesis in Kluyveromyces marxianus. ACS Synth. Biol. 7, 2647-2655. doi: 10.1021/acssynbio.8b00331

Mikkelsen, M. D., Buron, L. D., Salomonsen, B., Olsen, C. E., Hansen, B. G., Mortensen, U. H., et al. (2012). Microbial production of indolylglucosinolate through engineering of a multi-gene pathway in a versatile yeast expression platform. Metab. Eng. 14, 104-111. doi: 10.1016/j.ymben. 2012.01.006

Morrissey, J. P., Etschmann, M. M., Schrader, J., and de Billerbeck, G. M. (2015). Cell factory applications of the yeast Kluyveromyces marxianus for the biotechnological production of natural flavour and fragrance molecules. Yeast 32, 3-16. doi: 10.1002/yea.3054

Nambu-Nishida, Y., Nishida, K., Hasunuma, T., and Kondo, A. (2017). Development of a comprehensive set of tools for genome engineering in a coldand thermo-tolerant Kluyveromyces marxianus yeast strain. Sci. Rep. 7, 1-7. doi: 10.1038/s41598-017-08356-5

Ng, H., and Dean, N. (2017). Dramatic improvement of CRISPR/Cas9 editing in Candida albicans by increased single guide RNA expression. Mol. Biol. Physiol. 2, 1-17. doi: 10.1128/mSphere.00385-16

Obst, U., Lu, T. K., and Sieber, V. (2017). A modular toolkit for generating Pichia pastoris secretion libraries. ACS Synth. Biol. 6, 1016-1025. doi: 10.1021/acssynbio.6b00337

Ortiz-Merino, R. A., Varela, J. A., Coughlan, A. Y., Hoshida, H., da Silveira, W. B., Wilde, C., et al. (2018). Ploidy variation in Kluyveromyces marxianus separates dairy and non-dairy isolates. Front. Genet. 9, 1-16. doi: 10.3389/fgene.2018.00094

Prielhofer, R., Barrero, J. J., Steuer, S., Gassler, T., Zahrl, R., Baumann, K., et al. (2017). GoldenPiCS: a golden gate-derived modular cloning system for applied synthetic biology in the yeast Pichia pastoris. BMC Syst. Biol. 11, 1-14. doi: 10.1186/s12918-017-0492-3

Schabort, du. T. W., Letebele, P. K., Steyn, L., Kilian, S. G., and Du Preez, J. C. (2016). Differential RNA-seq, multi-network analysis and metabolic regulation analysis of Kluyveromyces marxianus reveals a compartmentalised response to xylose. PLOS ONE 11:e156242. doi: 10.1371/journal.pone.01 56242

Suzuki, A., Fujii, H., Hoshida, H., and Akada, R. (2015). Gene expression analysis using strains constructed by NHEJ-mediated one-step promoter cloning in the yeast Kluyveromyces marxianus. FEMS Yeast Res. 15, 1-10. doi: 10.1093/femsyr/fov059

Varela, J. A., Montini, N., Scully, D., Van der Ploeg, R., Oreb, M., Boles, E., et al. (2017). Polymorphisms in the LAC12 gene explain lactose utilisation variability in Kluyveromyces marxianus strains. FEMS Yeast Res. 17, 1-13. doi: 10.1093/femsyr/fox021

Varela, J. A., Puricelli, M., Montini, N., and Morrissey, J. P. (2019). Expansion and Diversification of MFS Transporters in Kluyveromyces marxianus. Front. Microbiol. 9:3330. doi: 10.3389/fmicb.2018.03330

Vyas, V. K., Barrasa, M. I., and Fink, G. R. (2015). A Candida albicans CRISPR system permits genetic engineering of essential genes and gene families. Sci. Adv. 1:e1500248. doi: 10.1126/sciadv.1500248

Wagner, J. M., and Alper, H. S. (2016). Synthetic biology and molecular genetics in non-conventional yeasts: current tools and future advances. Fungal Genet. Biol. 89, 126-136. doi: 10.1016/j.fgb.2015.12.001 
Weber, E., Engler, C., Gruetzner, R., Werner, S., and Marillonnet, S. (2011). A modular cloning system for standardized assembly of multigene constructs. PLoS ONE 6:e16765. doi: 10.1371/journal.pone.0016765

Wu, D., Topper, L. M., and Wilson, T. E. (2008). Recruitment and dissociation of nonhomologous end joining proteins at a DNA doublestrand break in Saccharomyces cerevisiae. Genetics 178, 1237-1249. doi: 10.1534/genetics.107.083535

Xie, S., Shen, B., Zhang, C., Huang, X., and Zhang, Y. (2014). SgRNAcas9: a software package for designing CRISPR sgRNA and evaluating potential offtarget cleavage sites. PLoS ONE 9:e100448. doi: 10.1371/journal.pone.0100448

Yang, C., Hu, S., Zhu, S., Wang, D., Gao, X., and Hong, J. (2015a). Characterizing yeast promoters used in Kluyveromyces marxianus. World J. Microbiol. Biotechnol. 31, 1641-1646. doi: 10.1007/s11274-015-1899-x

Yang, H., Matsumoto, Y., Trujillo, K. M., Lees-Miller, S. P., Osley, M. A., and Tomkinson, A. E. (2015b). Role of the yeast DNA repair protein Nej1 in end processing during the repair of DNA double strand breaks by non-homologous end joining. DNA Repair 31, 1-10. doi: 10.1016/j.dnarep.2015.04.003

Yarimizu, T., Nonklang, S., Nakamura, J., Tokuda, S., Nakagawa, T., Lorreungsil, S., et al. (2013). Identification of auxotrophic mutants of the yeast
Kluyveromyces marxianus by non-homologous end joining-mediated integrative transformation with genes from Saccharomyces cerevisiae. Yeast 30, 485-500. doi: 10.1002/yea.2985

Zhou, J., Zhu, P., Hu, X., Lu, H., and Yu, Y. (2018). Improved secretory expression of lignocellulolytic enzymes in Kluyveromyces marxianus by promoter and signal sequence engineering. Biotechnol. Biofuels 11:235. doi: 10.1186/s13068-018-1232-7

Conflict of Interest Statement: The authors declare that the research was conducted in the absence of any commercial or financial relationships that could be construed as a potential conflict of interest.

Copyright (C) 2019 Rajkumar, Varela, Juergens, Daran and Morrissey. This is an open-access article distributed under the terms of the Creative Commons Attribution License (CC BY). The use, distribution or reproduction in other forums is permitted, provided the original author(s) and the copyright owner(s) are credited and that the original publication in this journal is cited, in accordance with accepted academic practice. No use, distribution or reproduction is permitted which does not comply with these terms. 